\title{
Incremental ECAP with Converging Billets
}

\author{
Andrzej Rosochowski ${ }^{1, a}$, Lech Olejnik ${ }^{2, b}$ \\ ${ }^{1}$ Design, Manufacture and Engineering Management, University of Strathclyde, James Weir \\ Building, 75 Montrose Street, Glasgow G1 1XJ, United Kingdom \\ ${ }^{2}$ Institute of Manufacturing Processes, Warsaw University of Technology, ul. Narbutta 85, 02-524 \\ Warsaw, Poland \\ aa.rosochowski@strath.ac.uk, ${ }^{\mathrm{b}}$ I.olejnik@wip.pw.edu.pl
}

Keywords: severe plastic deformation, incremental forming, equal channel angular pressing, ultrafine grained metals.

\begin{abstract}
A new concept of incremental equal channel angular pressing (I-ECAP) with converging billets is proposed and simulated numerically. It follows a recently introduced process of ECAP with converging billets, in which the contact surface between converging billets plays the same role as a movable die wall in the output channel of classical ECAP and thus reduces friction and the process force. The process productivity is doubled and material pickup, especially problematic in the output channel, avoided. However, ECAP with converging billets, as any ECAP-based process, suffers from a limited length of the billets it can process. This paper proposes an incremental version of ECAP with converging billets, which enables processing very long billets. Additionally, a new option for ECAP or I-ECAP with converging billets is considered, which assumes their separation with a movable tool. This tool can also be used to apply a back force. FEM simulations of all these processes enable their comparison in terms of strain distribution and the force required.
\end{abstract}

\section{Introduction}

Bulk metals with ultrafine grained (UFG) structure, characterised by the average grain size <1 $\mu \mathrm{m}$, draw substantial attention due to their unique mechanical and physical properties. The preferable method of producing bulk UFG metals, which avoids technical problems and a health hazard associated with nanopowders, is severe plastic deformation (SPD) [1]. In this method, a very large plastic deformation (true strain $>3$, depending on the metal) "subdivides" coarse metal grains into sub-micrometre size grains. Unlike traditional metal forming processes, SPD processes retain the shape of the workpiece.

Equal channel angular pressing (ECAP), originally proposed by Segal et al. under the name of equal channel angular extrusion [2], is the most popular SPD process used to produce UFG metals. In this process (Fig. 1), a square or cylindrical billet is pushed by a punch through an input constant profile channel to an output channel of the same profile orientated at an angle $\geq 90^{\circ}$ to the input channel. Plastic deformation of the material is caused by simple shear in a thin layer along the diagonal plane at the channel crossing. The process is usually repeated several times with the billet being rotated about its axis between consecutive passes. The process is simple in terms of tooling and machines used, however, it suffers from friction present in the die channels. Friction increases the process force and tool contact pressure, limits the length of billets processed and causes material pickup. To reduce friction, a concept of movable die walls both in the input and the output channel of the die was proposed [3]. The least complicated seems to be a movable wall representing the bottom part of the output channel. But even in this case, the introduction of a movable die wall leads to a more complex and expensive ECAP device. Even if friction between a billet and the bottom part of the output channel is eliminated, it does not disappear completely; it is just moved to a new location between the stationary and movable device elements. Thus friction reduction in the output channel still remains a challenge. 


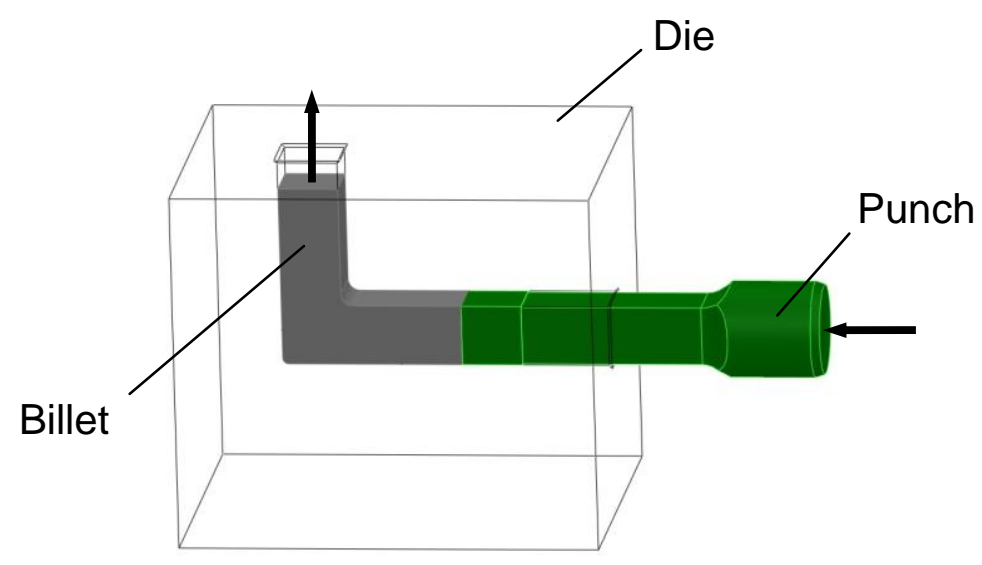

Fig. 1: Process/tool configuration in classical ECAP.

A new solution to the problem of friction in the output channel of an ECAP die has been proposed recently. It is based on the idea of ECAP with converging billets, which was explained and modelled using finite element (FE) simulation [4] as well as proven experimentally [5]. However, ECAP with converging billets, as any ECAP-based process, suffers from a limited length of the billets it can process. This paper proposes an incremental version of ECAP with converging billets, which enables processing very long billets. Additionally, a new option for ECAP or I-ECAP with converging billets is considered, which assumes their separation with a movable tool. This tool can also be used to apply a back force. FEM simulations of all these processes enable their comparison in terms of strain distribution and the force required.

\section{ECAP with converging billets}

In its basic configuration (Fig. 2), ECAP with converging billets uses two substantially equal square or rectangular input channels converging into a single output channel, which is twice as wide as the input channels so that it can accept two converging billets. The contact surface between converging billets plays the same role as a movable bottom wall in the output channel of classical ECAP mentioned above. It reduces friction and the process force. This effect is achieved without using a complex die with movable parts. Instead, two punches are used to push two billets synchronously from the opposite sides. The system doubles productivity compared to the case of processing a single billet.

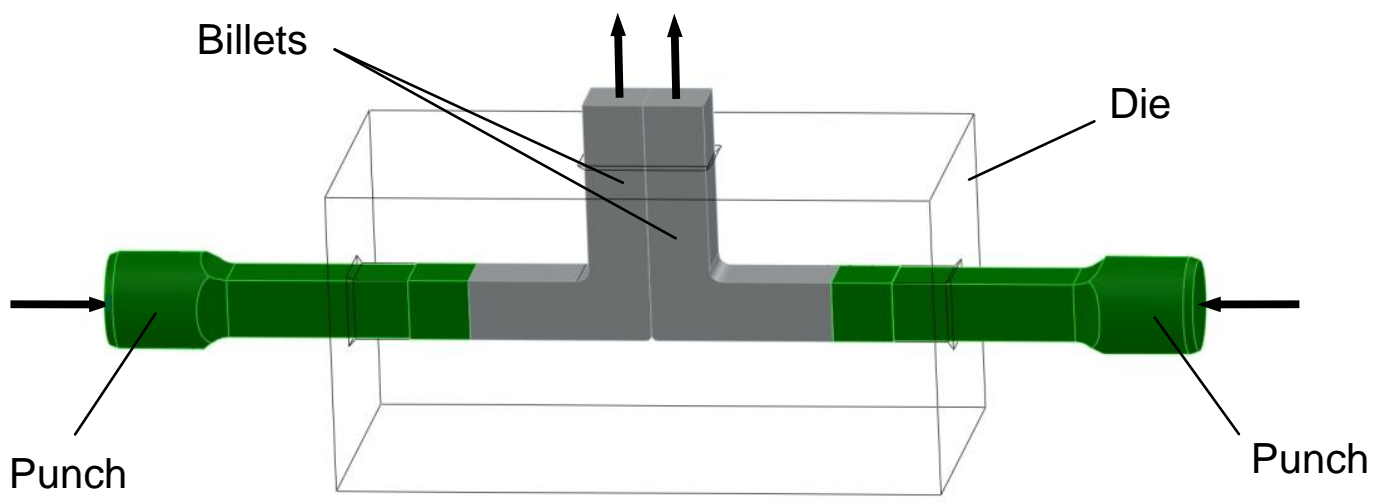

Fig. 2: Process/tool configuration for ECAP with two converging billets. 
Fig. 3 shows a comparison of classical ECAP of a single billet (without moving die walls) and ECAP with two converging billets in terms of strain distribution [5]. The finite element simulation (using Abaqus/Explicit ) was carried out for pure aluminium billets, with $8 \times 8 \mathrm{~mm}$ cross section. Strain appears to be similar, except the bottom part of the billet, where it is smaller for ECAP with converging billets. ECAP with converging billets reduces friction in the output channel, which leads to a lower value of the maximum process force [5]. For friction coefficient $\mu=0.1$, the force reduction predicted by plane strain FE simulation was $23 \%$ (Fig. 3).

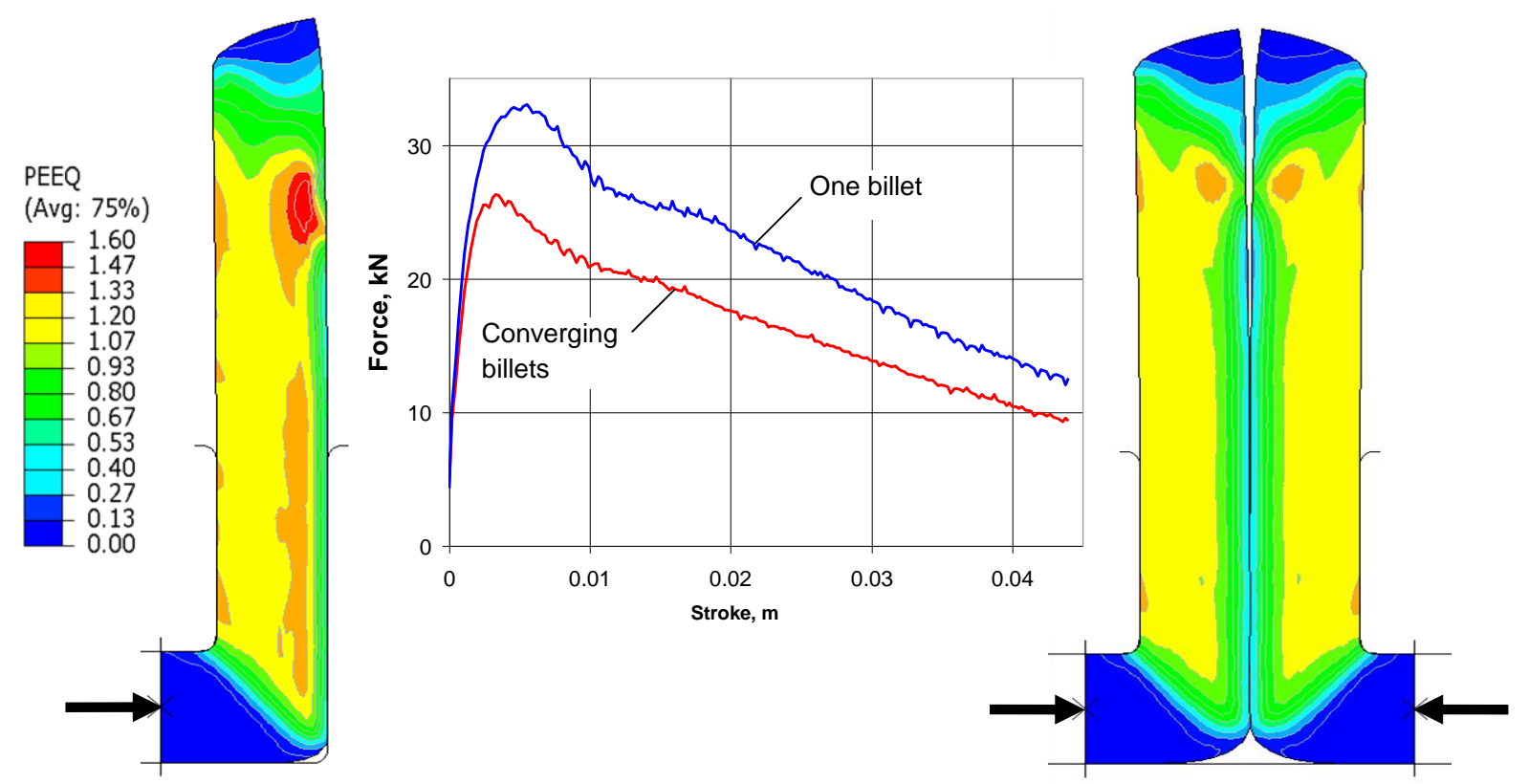

Fig. 3: Distribution of equivalent plastic strain in single-billet ECAP and ECAP with two converging billets and force history for both processes [5].

\section{Incremental ECAP with converging billets}

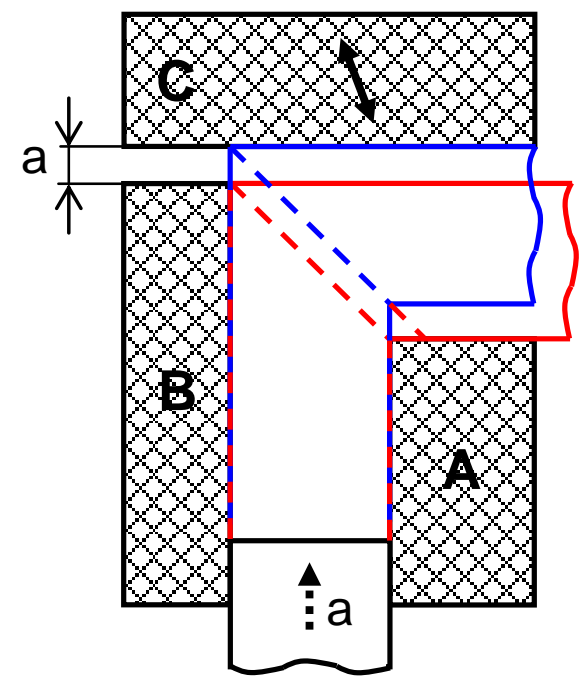

Fig. 4: Schematic of I-ECAP.
The basic version of Incremental ECAP (I-ECAP) is shown in Fig. 4 [6]. The die $\mathrm{C}$ is a working die, which moves in a reciprocating manner at an appropriate angle to the billet. Feeding of the billet takes place during withdrawal of that die. When feeding ends and the billet becomes fixed, the die approaches and deforms it plastically in a "dashed" zone (Fig. 4). The mode of deformation is that of simple shear and, provided the feeding stroke (distance "a" in Fig. 4) is not excessive, consecutive shear zones overlap resulting in a uniform strain distribution along the billet. Separation of the feeding and deformation stages reduces or eliminates friction in the input channel; this substantially reduces the feeding force and enables processing of very long/infinite billets. There have been a number of publications describing further developments of the method and experimental results, e.g. [7]. 


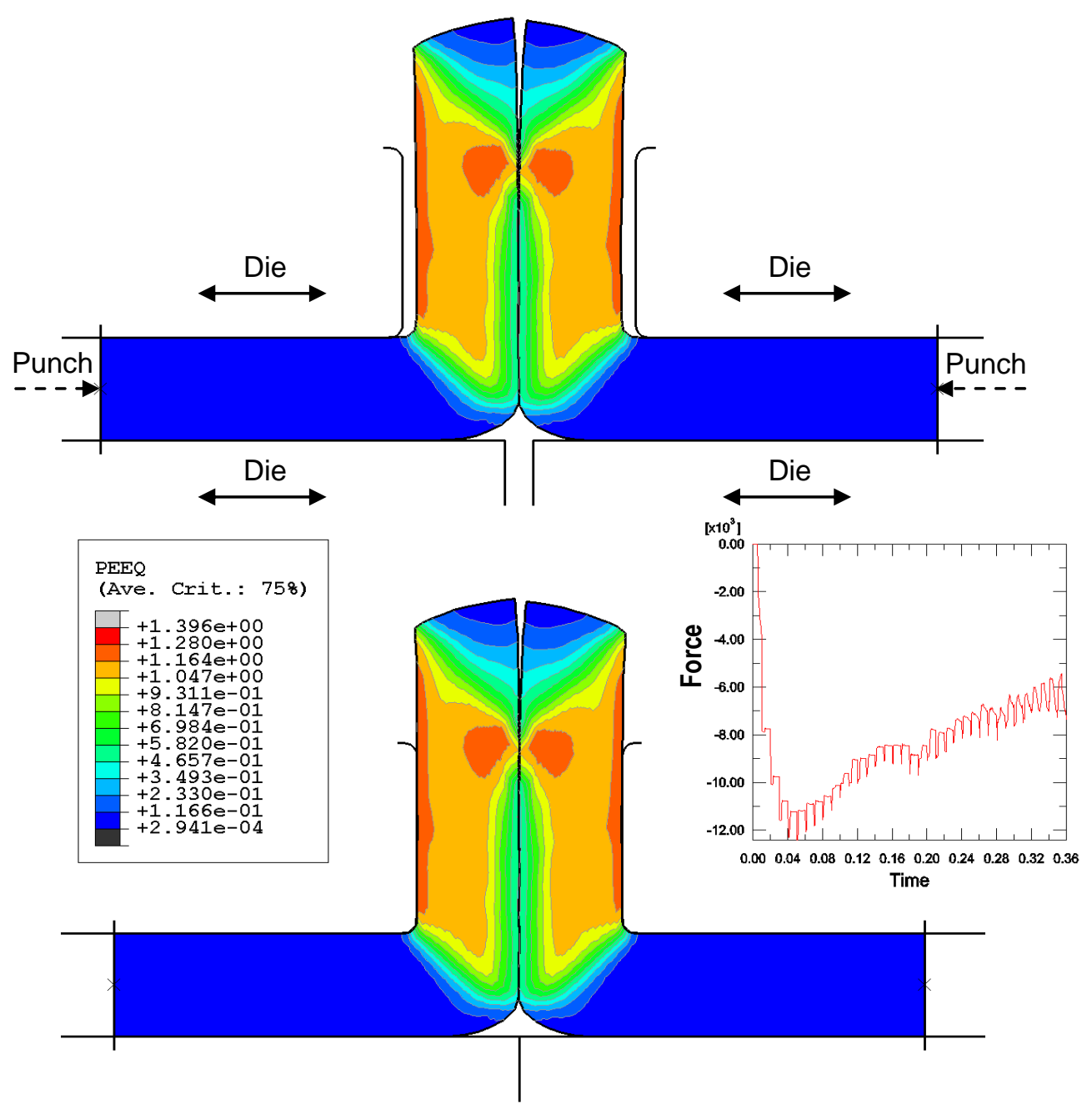

Fig. 5: Principle of I-CAP with converging billets; strain distribution and punch force.

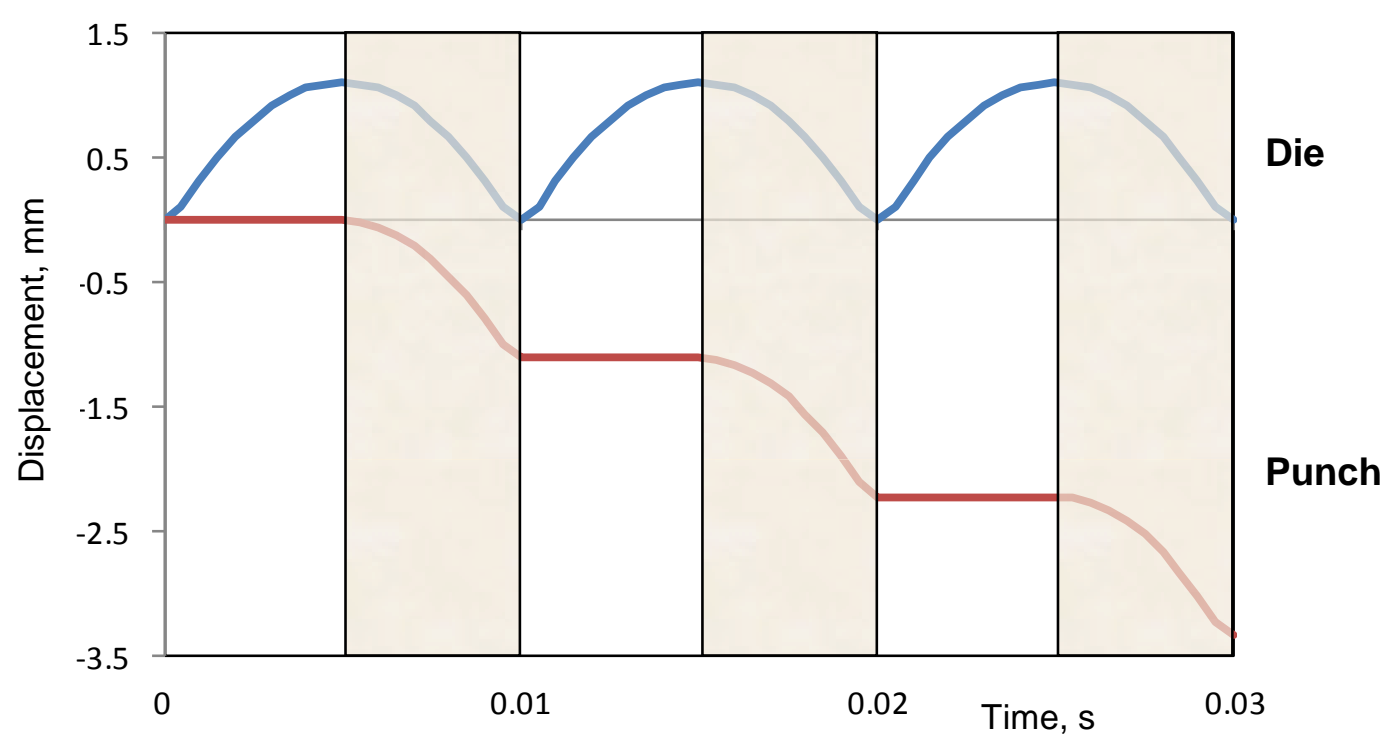

Fig. 6: Principle of I-CAP with converging billets; synchronisation of die and punch movement. 
I-ECAP with converging billets is different from the original version of I-ECAP presented in Fig. 4 because it swaps the movable tools. As a result of using converging billets, the die $\mathrm{C}$ does not exist but its role is assigned to the dies $\mathrm{A}$ and $\mathrm{B}$. Thus all the dies follow a reciprocating movement while punches serve as incremental feeders (Fig. 5). Fig. 6 helps understand the kinematics of the tools with the shaded areas indicating common movement of the dies and the punches; outside the shaded areas the dies move backwards while punches are fixed. In this way, friction in the input channels can be reduced as indicated by a much smaller punch force required for this process compared to ECAP with converging billets (13 kN vs. $26 \mathrm{kN})$.

However, both processes can potentially suffer from an unstable flow of the material if there is a lack of symmetry in billet dimensions or properties. Also, strain at the billet's interface is lower compared to classical ECAP. These can be rectified by using a separating tool and applying a back force to it.

\section{Adding separating tool}
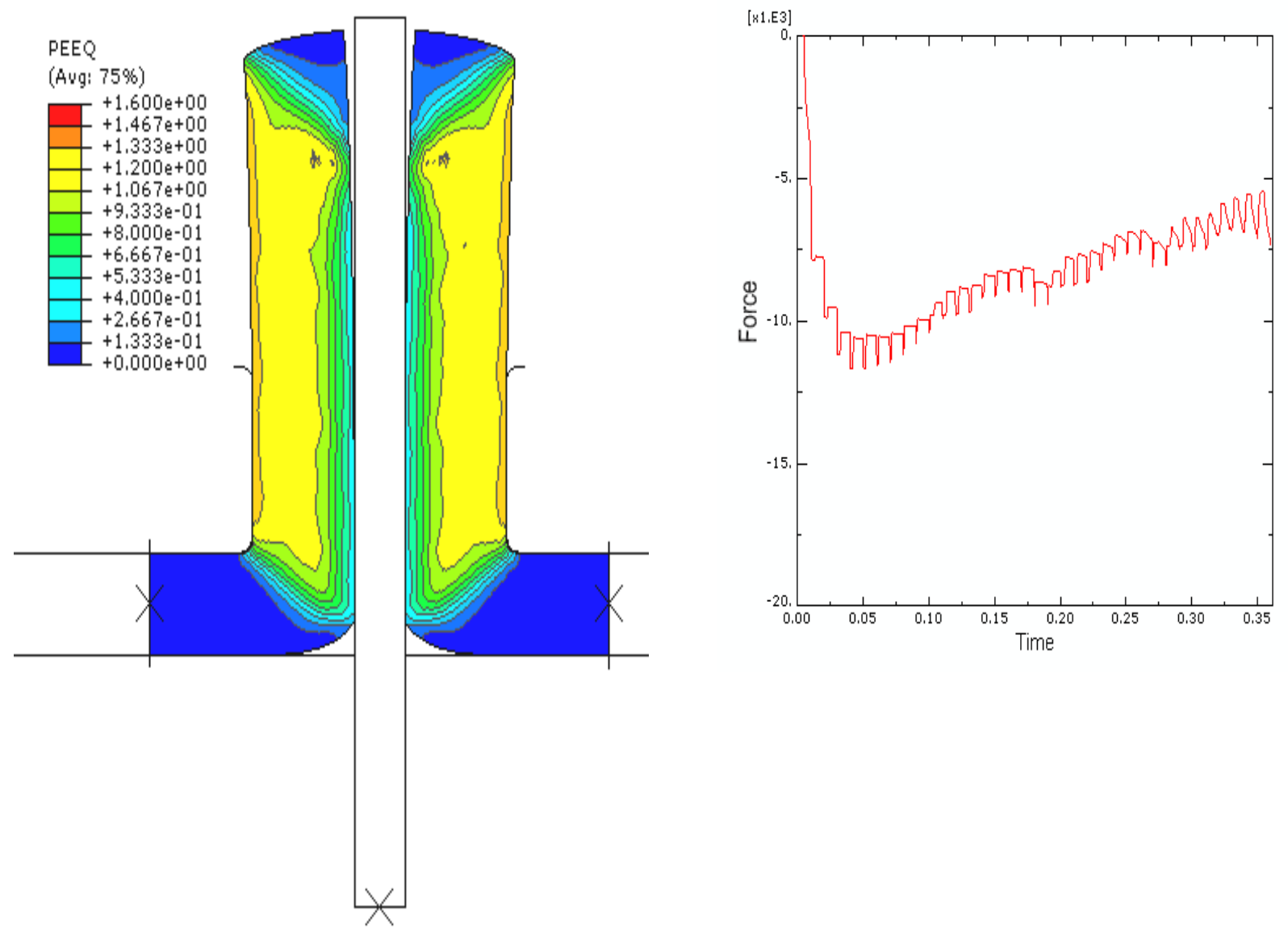

Fig. 7: I-CAP with converging billets and a separating tool: strain distribution (a) and punch force (b).

The separating tool, shown in Fig. 7, can be a bar or plate put between the two billets in the output channel and moving freely with the billets. Since it does not slide against the billets, there is no friction. Thus the punch force remains the same as in the case of billets in contact $(13 \mathrm{kN})$. If the separating tool is shaped as shown in Fig. 8, it can be used to apply a back force to the ends of billets. This force could be applied either as a compression force at the front end of the separating tool so its stem would be unloaded or as a tensile force pulling the stem. By applying a back force of $3 \mathrm{kN}$, the punch force increases from $13 \mathrm{kN}$ to $18 \mathrm{kN}$, but strain distribution is greatly improved. 

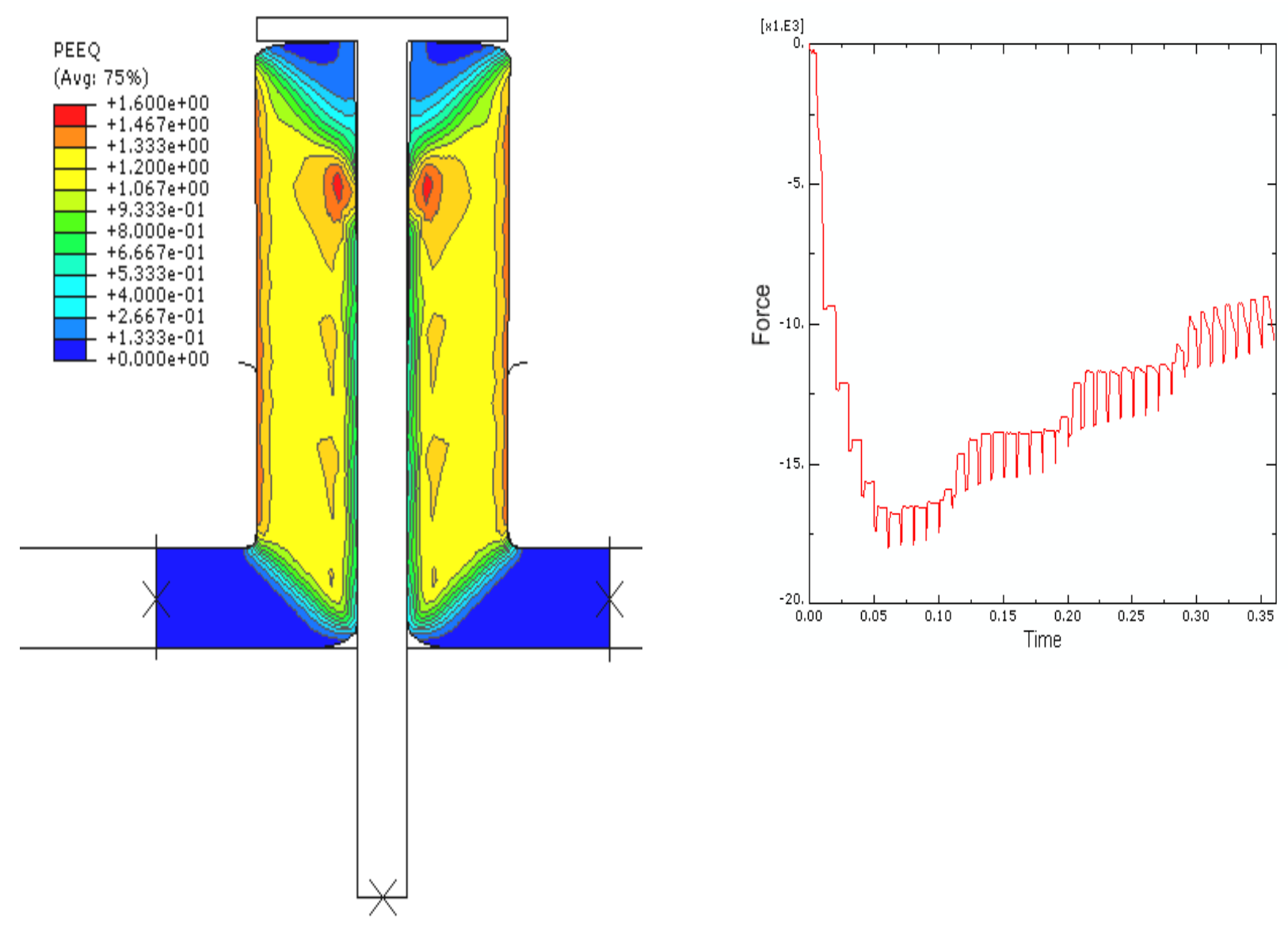

Fig. 8: I-CAP with converging billets and back force of $3 \mathrm{kN}$ applied using separating tool: strain distribution (a) and punch force (b).

\section{Discussion and conclusions}

The new proposed process of I-ECAP with converging billets has two main advantages over its non-incremental version. The first one is substantial reduction of the force required to feed/deform the material. Fig. 9 illustrates this point by comparing punch forces in different processes. While ECAP with converging billets reduces the punch force by $23 \%$ compared to classical ECAP, IECAP with converging billets reduces that force by another $50 \%$ (all results for Coulomb friction coefficient of 0.1). Adding a back force to the separating tool increases the punch force. The back force is limited by the yield force of the billets leaving the output channel or their buckling force.

Another advantage of the incremental process is the possibility of processing long billets. However, this would require abandoning punches and allowing the dies to clamp/unclamp the billets according to the phase of the cycle. Also applying the back force could be problematic for very long billets.

The main conclusions can be formulated as follows:

(1) A new original process of I-ECAP with converging billets has been proposed. It is a combination of ECAP with converging billets and incremental ECAP, in which the dies follow a reciprocating movement while punches feed the billets incrementally in a synchronised way.

(2) The new process reduces the punch force by $50 \%$ compared to the non-incremental version of ECAP with converging billets (for friction coefficient of 0.1 ). 
(3) With a separating tool, it is possible to add a back force to the billets. This increases the punch force but results in more uniform strain distribution and possible less damage to the material caused by hydrostatic tension.

(4) Because of the incremental nature of the process, there is a possibility of processing long billets. However, this would require the die kinematics to become quite complex; the reciprocating movement would have to be combined with the clamping and unclamping action of the dies.

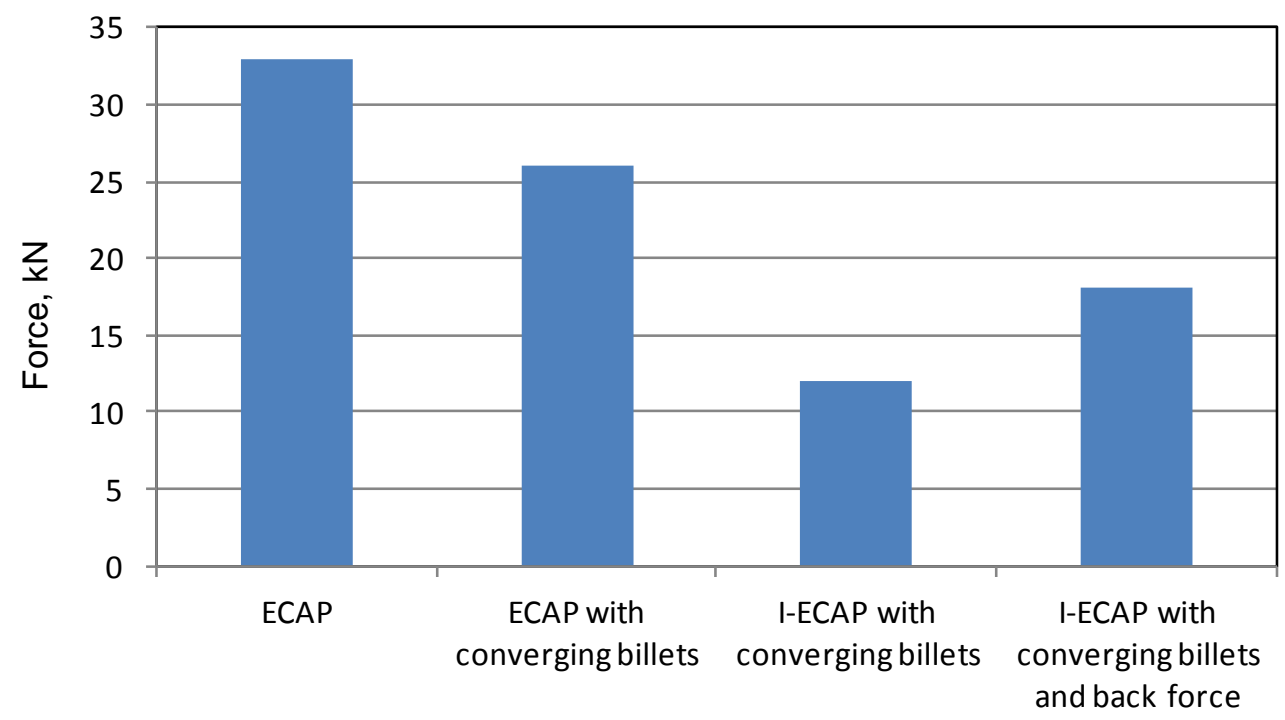

Fig. 9: Comparison of punch force for different versions of ECAP and I-ECAP.

Acknowledgements Part of this research was supported by the Engineering and Physical Sciences Research Council [grant number EP/G03477X/1].

\section{References}

[1] A. Azushima, R. Kopp, A. Korhonen, D.Y. Yang, F. Micari, G.D. Lahoti, P. Groche, J. Yanagimoto, N. Tsuji, A. Rosochowski, A. Yanagida, CIRP Annals - Manufacturing Technology 57 (2008) 716-735.

[2] V.M. Segal, V.A. Reznikov, A.E. Drobyshevskiy, V.I. Kopylov, Russian Metallurgy 1 (1981) 99-105.

[3] V.M. Segal, Materials Science and Engineering A 386 (2004) 269-276.

[4] A. Rosochowski, L. Olejnik, in: G. Hirt, A.E. Tekkaya (Eds), Proc. of the $10^{\text {th }}$ International Conference on Technology of Plasticity, ICTP 2011, 25-30 September 2011, Aachen, Germany, pp. 235-240.

[5] A. Rosochowski, L. Olejnik, J. Richert, M. Rosochowska, M. Richert, Materials Science and Engineering A 560 (2013) 358-364.

[6] A. Rosochowski, L. Olejnik, in: E. Cueto, F. Chinesta (Eds), Proc. of the 10th International Conference on Material Forming, Esaform 2007, April 18-20, 2007, Zaragoza, Spain, American Institute of Physics, Proceedings 907, 2007, p. 653.

[7] A. Rosochowski, L. Olejnik, Materials Science Forum 674 (2011) 19-28. 